Bull. Korean Math. Soc. 49 (2012), No. 6, pp. 1179-1192

http://dx.doi.org/10.4134/BKMS.2012.49.6.1179

\title{
DOUBLY NONLINEAR PARABOLIC EQUATIONS INVOLVING $p$-LAPLACIAN OPERATORS VIA TIME-DISCRETIZATION METHOD
}

\author{
Kiyeon Shin and Sujin Kang
}

\begin{abstract}
In this paper, we consider a doubly nonlinear parabolic partial differential equation $\frac{\partial \beta(u)}{\partial t}-\Delta_{p} u+f(x, t, u)=0$ in $\Omega \times[0, T]$, with Dirichlet boundary condition and initial data given. We prove the existence of a discrete approximate solution by means of the Rothe discretization in time method under some conditions on $\beta, f$ and $p$.
\end{abstract}

\section{Introduction}

In this paper, we study a doubly nonlinear parabolic partial differential equation involving the $p$-Laplacian operator. More precisely, we are interested in the existence and uniqueness of the solution of problem

$$
\begin{cases}\frac{\partial \beta(u)}{\partial t}-\Delta_{p} u+f(x, t, u)=0 & \text { in } \Omega \times[0, T], \\ u=0 & \text { on } \partial \Omega \times[0, T], \\ u(\cdot, 0)=u^{0} & \text { in } \Omega,\end{cases}
$$

where $\Delta_{p} u=\operatorname{div}\left(|\nabla u|^{p-2} \nabla u\right), 1<p<\infty, \beta$ is a nonlinearity of porous medium type and $f$ is a nonlinearity of reaction diffusion type. Let $\Omega$ be a regular open bounded subset of finite dimensional space $\mathbb{R}^{d}(d \geq 3)$ and $\partial \Omega$ be its smooth boundary. These problems arise in many applications in the fields of mechanics, physics and biology (non Newtonian fluids, gas flow in porous media, spread of biological populations, etc).

Equations of the form (1) for $p=2$ has been motivated by the following two papers. The first one due to M. Gurtin [9] gives a non phenomenological derivation of the generalized Allen-Cahn equation. This equation describes some particular aspects of isothermal phase separation process. Performing a clear distinction between thermodynamical laws and constitutive equations, which is in the first part of [9], M. Gurtin propose the following generalization

Received July 13, 2009.

2010 Mathematics Subject Classification. 35K55, 35K45.

Key words and phrases. doubly nonlinear, $p$-Laplacian, Rothe method. 
of the Allen-Cahn equation

$$
a\left(u, \nabla u, u_{t}\right) u_{t}-\Delta u+f(t, x, u)=0
$$

with $a \geq 0$. So, this equation can be degenerate and if the coefficient $a$ depends only on $u$, we may rewrite (2) under the form of equation (1) for $p=2$. Next, in [13], A. Miranvile and G. Schimperna use Gurtin's approach to model nonisothermal phase transition problem. They end up with the following system of PDE's

$$
\left(u^{2}\right)_{t}-\Delta u=f+u \chi \chi_{t}+\left(\chi_{t}\right)^{2}, \quad \chi_{t}-\Delta \chi+g(\chi)=-u \chi,
$$

where the unknowns are the absolute temperature $u$ and the phase field $\chi$. If $\chi$ is given, then the above equation takes the form (1) if $\beta(u)=u^{2}$ for positive $u$. Regarding the potential $f$, we assume that it satisfies some sign condition which is often used in phase transition problems [7]. For instance, $f(t, x, u)$ may be equal to $f(u)=u^{p}-u^{2}$ with $p \geq 3$ odd. Uniqueness of the solution to (2) for $p=2$ satisfying homogeneous Dirichlet boundary conditions is a simple consequence of a deep result of Otto [14].

In case of $p=2$ of (1), M. Schatzman, A. Eden, B. Michaux, J. M. Rakotoson, A. Rougirel, J. I. Diaz and J. F. Padial [4, 5, 6, 15, 17] dedicated to the existence of solutions and to the large time behavior of these equations in a lot of works. M. Schatzman [17] considered for the problem in which $\beta(u)=u$ for $p=2$ of (1) and then the problem reduces the reaction-diffusion equations. A. Eden, B. Michaux and J. M. Rakotoson studied the existence of solutions using the method of semi-discretization [5] as well as the method of Galerkin approximation [6]. A. Rougirel [15] studied the solution of asymptotic behavior for $\left|\frac{\partial f}{\partial t}(t, x, u)\right| \leq C_{M},\left(C_{M}>0\right)$. And J. I. Diaz and J. F. Padial [4] are considered the existence of solution in $B V_{t}(Q)$ space using $\beta\left(u_{t}\right)$ instead of $\frac{\partial \beta(u)}{\partial t}$.

In case of $p>1$ of (1), A. Bensoussan, L. Boccardo and F. Murat [3] studied the existence of solution for $\beta=0$ and A. El Hachimi and H. El Ouardi [8] studied the existence and regularity of this equation under the fact that $f$ is differentiable by the method of Galerkin approximation.

Various abstract evolution equations have been considered using Rothe timediscretization method, see, for instance, Kartsatos and Parrott [10] and references cited therein. In the paper, the authors solved the initial-boundary value problem for the time-dependent functional equation by the time-discretization method.

This is the plan of paper. We recall our assumptions and state main results in Section 2. In Section 3, we show the existence of discrete scheme. And, after showing some estimates on the approximations, the passage to the limit and the existence results are given in Section 4. 


\section{Assumptions and main results}

We let $\|\cdot\|_{p},\|\cdot\|_{1, p}$ and $\|\cdot\|_{-1, p}$ denote the norm in $L^{p}(\Omega), W_{0}^{1, p}(\Omega)$ and $W^{-1, p}(\Omega)$ for $1<p<\infty$, respectively. And $\langle\cdot, \cdot\rangle$ denotes the duality between $W_{0}^{1, p}(\Omega)$ and $W^{-1, p}(\Omega)$ or denotes inner product of $L^{2}(\Omega)$. For $1<p<\infty$, we define the conjugate $p^{\prime}$ of $p$ by $1 / p+1 / p^{\prime}=1$. In this paper, $C_{i}$ and $C$ will denote positive constants and $\lambda_{i}$ the imbedding constants such that

(cf. [1]).

$$
\begin{cases}\|\cdot\|_{p} \leq \lambda_{1}\|\cdot\|_{1, p} & \text { if } \quad \frac{2 d}{2+d} \leq p<\infty \\ \|\cdot\|_{2} \leq \lambda_{2}\|\cdot\|_{1, p} & \text { if } \quad \frac{2 d}{2+d} \leq p<2 \\ \|\cdot\|_{1} \leq \lambda_{3}\|\cdot\|_{p} \leq \lambda_{4}\|\cdot\|_{1, p} & \text { if } \quad p \geq 2\end{cases}
$$

We define $\psi$ by $\psi(t)=\int_{0}^{t} \beta(s) d s$ for $t \in \mathbb{R}$ and a continuous function $\beta$ with $\beta(0)=0$. Then, the Legendre transform of $\psi$ is also defined by $\psi^{*}(\tau)=$ $\sup _{s \in \mathbb{R}}\{\tau s-\psi(s)\}$.

Now, we present our assumptions which are used throughout this paper.

We suppose $d^{*} \leq p<\infty$ where $d^{*}=(2 d) /(d+2), u^{0} \in L^{\infty}(\Omega)$ with $u^{0}=0$ on $\partial \Omega$ and the followings:

(H1) The function $\beta: \mathbb{R} \rightarrow \mathbb{R}$ is increasing and continuous with $\beta(0)=0$.

(H2) For $\xi \in \mathbb{R}$, the map $(x, t) \mapsto f(x, t, \xi)$ is measurable and $\xi \mapsto f(x, t, \xi)$ is continuous a.e. in $\Omega \times[0, T]$. Furthermore, we assume that there exits $C_{1}>0$ such that $\operatorname{sign} \xi f(x, t, \xi) \geq-C_{1}$ for a.e. $(x, t) \in \Omega \times[0, T]$.

(H3) For all $M>0$, there exists $C_{M}>0$ such that, if $|\xi|+\left|\xi^{\prime}\right| \leq M$, then

$$
\left|f(x, t, \xi)-f\left(x, t, \xi^{\prime}\right)\right|^{\alpha} \leq C_{M}\left(\beta(\xi)-\beta\left(\xi^{\prime}\right)\right)\left(\xi-\xi^{\prime}\right),
$$

where $\alpha= \begin{cases}2 & \text { if } 1<p<2, \\ p^{\prime} & \text { if } \quad p \geq 2 .\end{cases}$

(H3) $)^{\prime}$ There is $C_{2}>0$ such that $\xi \mapsto f(x, t, \xi)+C_{2} \beta(\xi)$ is increasing for almost $(x, t) \in \Omega \times[0, T]$.

(H4) For almost every $x \in \Omega$ and for all $M>0$, there exists $\widetilde{C}_{M}>0$ such that, if $t+t^{\prime}+|\xi| \leq M$, then

$$
\left|f(x, t, \xi)-f\left(x, t^{\prime}, \xi\right)\right| \leq \widetilde{C}_{M}\left|t-t^{\prime}\right|^{1 / \alpha}
$$

where $\alpha$ is same as in (H3).

Definition ([2]). Let $X$ be a reflexive Banach space and $A: X \rightarrow X^{\prime}$. We say that $A$ is monotone if $\langle A y-A z, y-z\rangle \geq 0$ for all $y, z \in X$, and $A$ is hemicontinuous if for each $y, z, w \in X$ the real-valued function $t \rightarrow\langle A(y+$ $t z), w\rangle$ is continuous.

Lemma 2.1 (Minty's Theorem [18]). Let $X$ be a reflexive Banach space. If $A: X \rightarrow X^{\prime}$ is monotone and hemicontinuous, then

$$
A y=f \quad \text { if and only if } \quad\langle f-A z, y-z\rangle \geq 0
$$

for all $z \in X$. 
Lemma $2.2([3])$. Let $\Omega$ be a bounded set in $\mathbb{R}^{d}$. Let $1<p<\infty$ be fixed and $A: W_{0}^{1, p}(\Omega) \rightarrow W^{1, p^{\prime}}(\Omega)$ a nonlinear operator defined by

$$
A(u)=-\operatorname{div} a(x, u, D u),
$$

where $a(x, s, \xi)$ is a Carathéodory function $a: \Omega \times \mathbb{R} \times \mathbb{R}^{d} \rightarrow \mathbb{R}^{d}$ such that

$$
\begin{aligned}
& |a(x, s, \xi)| \leq \beta\left[|s|^{p-1}+|\xi|^{p-1}+k(x)\right], \\
& {[a(x, s, \xi)-a(x, s, \eta)](\xi-\eta)>0, \quad \xi \neq \eta,} \\
& a(x, s, \xi) \xi \geq \alpha|\xi|^{p},
\end{aligned}
$$

where $k(x) \in L^{p^{\prime}}(\Omega), k \geq 0, \beta>0$ and $\alpha>0$.

Let $g(x, s, \xi)$ be a Carathéodory function such that

$$
g(x, s, \xi) s \geq 0, \quad|g(x, s, \xi)| \leq b(|s|)\left(|\xi|^{p}+c(x)\right),
$$

where $b$ is a continuous and increasing function with (finite) values on $\mathbb{R}^{+}$and $c \in L^{1}(\Omega), c \geq 0$. Then, for $h \in W^{-1, p^{\prime}}(\Omega)$, the problem

$$
A u+g(x, u, \nabla u)=h,
$$

has at least one solution $u \in W_{0}^{1, p}(\Omega)$.

Lemma $2.3([16])$. If $u \in W_{0}^{1, p}(\Omega)$ is a solution to the equation

$$
-\Delta_{p} u+F(x, u)=h
$$

where $h \in W^{-1, r}, r>\frac{d}{p-1}$ and $F$ satisfies $\xi F(x, \xi) \geq 0$ in $\Omega \times \mathbb{R}$, then $u \in L^{\infty}(\Omega)$.

Now, we state our main results as follows:

Theorem 2.4. Under assumptions (H1), (H2), (H3) (or $\left.(\mathrm{H} 3)^{\prime}\right)$ and $(\mathrm{H} 4)$, there exists a unique solution $u$ of (1) such that

$$
\begin{cases}u \in L^{p}\left(0, T ; W_{0}^{1, p}(\Omega)\right) \cap L^{\infty}\left(0, T ; L^{\infty}(\Omega)\right) & \text { if } p \geq 2 \\ u \in L^{2}\left(0, T ; L^{2}(\Omega)\right) & \text { if } d^{*} \leq p<2 .\end{cases}
$$

\section{Existence of scheme}

For the problem (1), we consider the discrete scheme (DS) for $i=1,2, \ldots, N$,

$$
\begin{cases}\frac{\beta\left(u_{i}\right)-\beta\left(u_{i-1}\right)}{\tau}-\Delta_{p} u_{i}+f\left(x, i \tau, u_{i}\right)=0 & \text { in } \Omega, \\ u_{i}=0 & \text { in } \partial \Omega, \\ u_{0}=u^{0} & \text { in } \Omega,\end{cases}
$$

where $N \tau=T$ and $T$ is a fixed positive real. We shall show that (DS) has a solution $u_{i}$ for $i=1,2, \ldots, N$.

Theorem 3.1. Let (H1)-(H3) hold. Then for $i=1,2, \ldots, N$, there exists a unique solution $u_{i} \in W_{0}^{1, p}(\Omega) \cap L^{\infty}(\Omega)$ of (DS) for sufficiently small $\tau$. 
Proof. First of all, we rewrite (DS) as

$$
-\tau \Delta_{p} u_{i}+F\left(x, u_{i}\right)=\varphi_{i-1},
$$

where $F\left(x, u_{i}\right)=\beta\left(u_{i}\right)+\tau f\left(x, i \tau, u_{i}\right)+\tau C_{1} \operatorname{sign}\left(u_{i}\right)$ and $\varphi_{i-1}=\beta\left(u_{i-1}\right)+$ $\tau C_{1} \operatorname{sign}\left(u_{i}\right)$.

Now, we consider the equation

$$
-\tau \Delta_{p} u+F(x, u)=\varphi_{0}=\beta\left(u_{0}\right)+\tau C_{1} \operatorname{sign}(u),
$$

where $F(x, u)=\beta(u)+\tau f(x, \tau, u)+\tau C_{1} \operatorname{sign}(u)$ for fixed $\tau=T / N$. It is obvious that $a(x, u, D u):=|\nabla u|^{p-2} \nabla u$ satisfies all the three conditions of $a$ in Lemma 2.2 (cf [11]). In particular, we used the inequality $|a|^{p-2} a(a-b) \geq \frac{1}{p}|a|^{p}-\frac{1}{p}|b|^{p}$ for the second condition. Since $\beta$ is continuous, $\varphi_{0} \in L^{\infty}(\Omega)$. And, by (H1) and $(\mathrm{H} 2), g(x, u, \nabla u):=F(x, u)$ is a Carathéodory function with $u F(x, u) \geq 0$. Also, by $(\mathrm{H} 2),|F(x, u)| \leq \beta(|u|)+2 \tau C_{1}$. Thus, all the conditions of $g$ in Lemma 2.2 are satisfied. Therefore, there exists a solution $u \in W_{0}^{1, p}(\Omega)$ of (3). Moreover, by Lemma 2.3, $u \in W_{0}^{1, p}(\Omega) \cap L^{\infty}(\Omega)$. We put $u_{1}:=u$ and consider the equation $-\tau \Delta_{p} u+F(x, u)=\varphi_{1}=\beta\left(u_{1}\right)+\tau C_{1} \operatorname{sign}(u)$ where $F(x, u)=\beta(u)+\tau f(x, 2 \tau, u)+\tau C_{1} \operatorname{sign}(u)$. Continuing this process, we have a solution $u_{i}$ of (DS) for $i=1,2, \ldots, N$ such that $u_{i} \in W_{0}^{1, p}(\Omega) \cap L^{\infty}(\Omega)$ $(i=1,2, \ldots, N)$.

Next, we show the uniqueness of $u_{i}(i=1,2, \ldots, N)$. Let $u_{i}$ and $u_{i}^{*}$ be two solutions of (DS) for $i=1,2, \ldots, N$. Using the result which we will establish below (see Theorem 3.3), we have

$$
\left\|u_{i}\right\|_{1, p}+\left\|u_{i}^{*}\right\|_{1, p} \leq M
$$

where $M$ is a suitable positive constant independent of $\tau$. And, from [11] we have

$$
\left\langle-\Delta_{p} u+\Delta_{p} v, u-v\right\rangle \geq \begin{cases}C_{p}\|u-v\|_{1, p}^{p} & \text { if } \quad p \geq 2, \\ C_{p} \frac{\|u-v\|_{1, p}^{2}}{\left(\|u\|_{1, p}+\|v\|_{1, p}\right)^{2-p}} & \text { if } \quad 1<p<2,\end{cases}
$$

for all $u, v \in W_{0}^{1, p}(\Omega)$.

Since $u_{i}$ and $u_{i}^{*}$ are solutions of (DS), we have

$$
-\tau \Delta_{p} u_{i}+\tau \Delta_{p} u_{i}^{*}+\beta\left(u_{i}\right)-\beta\left(u_{i}^{*}\right)+\tau f\left(x, i \tau, u_{i}\right)-\tau f\left(x, i \tau, u_{i}^{*}\right)=0 .
$$

Multiplying (5) by $u_{i}-u_{i}^{*}$ and integrating over $\Omega$, we get

(6) $\left\langle-\tau \Delta_{p} u_{i}+\tau \Delta_{p} u_{i}^{*}, u_{i}-u_{i}^{*}\right\rangle+\tau \int_{\Omega}\left(f\left(x, i \tau, u_{i}\right)-f\left(x, i \tau, u_{i}^{*}\right)\right)\left(u_{i}-u_{i}^{*}\right) d x$

$$
+\int_{\Omega}\left(\beta\left(u_{i}\right)-\beta\left(u_{i}^{*}\right)\right)\left(u_{i}-u_{i}^{*}\right) d x=0 .
$$

(a) Suppose $p \geq 2$. By (4), the equation (6) becomes

$$
\tau C_{p}\left\|u_{i}-u_{i}^{*}\right\|_{1, p}^{p}+\int_{\Omega}\left(\beta\left(u_{i}\right)-\beta\left(u_{i}^{*}\right)\right)\left(u_{i}-u_{i}^{*}\right) d x
$$




$$
\leq \int_{\Omega}\left[\frac{1}{C_{M}^{1 / p^{\prime}}}\left(f\left(x, i \tau, u_{i}\right)-f\left(x, i \tau, u_{i}^{*}\right)\right)\right]\left(-C_{M}^{1 / p^{\prime}} \tau\left(u_{i}-u_{i}^{*}\right)\right) d x .
$$

By Young's inequality and (H3),

$$
\begin{aligned}
& \tau C_{p}\left\|u_{i}-u_{i}^{*}\right\|_{1, p}^{p}+\int_{\Omega}\left(\beta\left(u_{i}\right)-\beta\left(u_{i}^{*}\right)\right)\left(u_{i}-u_{i}^{*}\right) d x \\
\leq & \frac{1}{p^{\prime}} \int_{\Omega}\left(\beta\left(u_{i}\right)-\beta\left(u_{i}^{*}\right)\right)\left(u_{i}-u_{i}^{*}\right) d x+\frac{1}{p} \tau^{p} \lambda_{1} C_{M}^{\frac{p}{p^{\prime}}}\left\|u_{i}-u_{i}^{*}\right\|_{1, p}^{p} .
\end{aligned}
$$

Since $\beta$ is increasing, $0 \leq\left(\tau^{p} \lambda_{1} C_{M}^{p / p^{\prime}}-p \tau C_{p}\right)\left\|u_{i}-u_{i}^{*}\right\|_{1, p}^{p}$. It implies that for sufficiently small $\tau$, i.e., $\tau<\left(\frac{p C_{p}}{\lambda_{1} C_{M}^{p / p^{\prime}}}\right)^{1 /(p-1)}, u_{i}=u_{i}^{*}$ holds.

(b) Suppose $d^{*} \leq p<2$. Since $\left\|u_{i}\right\|_{1, p}+\left\|u_{i}^{*}\right\|_{1, p} \leq M$, by (H3) the equation (6) becomes

$$
\begin{aligned}
& \tau C_{p} \frac{\left\|u_{i}-u_{i}^{*}\right\|_{1, p}^{2}}{\left(\left\|u_{i}\right\|_{1, p}+\left\|u_{i}^{*}\right\|_{1, p}\right)^{2-p}}+\int_{\Omega}\left(\beta\left(u_{i}\right)-\beta\left(u_{i}^{*}\right)\right)\left(u_{i}-u_{i}^{*}\right) d x \\
\leq & -\tau \int_{\Omega}\left(f\left(x, i \tau, u_{i}\right)-f\left(x, i \tau, u_{i}^{*}\right)\right)\left(u_{i}-u_{i}^{*}\right) d x \\
\leq & \int_{\Omega} \frac{1}{2 C_{M}}\left|f\left(x, i \tau, u_{i}\right)-f\left(x, i \tau, u_{i}^{*}\right)\right|^{2} d x+\int_{\Omega} \frac{\tau^{2} C_{M}}{2}\left|u_{i}-u_{i}^{*}\right|^{2} d x \\
\leq & \frac{1}{2} \int_{\Omega}\left(\beta\left(u_{i}\right)-\beta\left(u_{i}^{*}\right)\right)\left(u_{i}-u_{i}^{*}\right) d x+\int_{\Omega} \frac{\tau^{2} C_{M}}{2}\left|u_{i}-u_{i}^{*}\right|^{2} d x .
\end{aligned}
$$

Therefore,

$$
\begin{aligned}
& \tau C_{p} \frac{1}{M^{2-p}}\left\|u_{i}-u_{i}^{*}\right\|_{1, p}^{2}+\int_{\Omega}\left(\beta\left(u_{i}\right)-\beta\left(u_{i}^{*}\right)\right)\left(u_{i}-u_{i}^{*}\right) d x \\
\leq & \frac{1}{2} \int_{\Omega}\left(\beta\left(u_{i}\right)-\beta\left(u_{i}^{*}\right)\right)\left(u_{i}-u_{i}^{*}\right) d x+\frac{\tau^{2} C_{M} \lambda_{2}}{2}\left\|u_{i}-u_{i}^{*}\right\|_{1, p}^{2} .
\end{aligned}
$$

In other words, since $\beta$ is increasing

$$
0 \leq \frac{1}{2} \int_{\Omega}\left(\beta\left(u_{i}\right)-\beta\left(u_{i}^{*}\right)\right)\left(u_{i}-u_{i}^{*}\right) d x \leq\left(\frac{C_{M} \tau^{2} \lambda_{2}}{2}-\frac{\tau C_{p}}{M^{2-p}}\right)\left\|u_{i}-u_{i}^{*}\right\|_{1, p}^{2} .
$$

Hence,

$$
0 \leq\left(\frac{C_{M} \tau^{2} \lambda_{2}}{2}-\frac{\tau C_{p}}{M^{2-p}}\right)\left\|u_{i}-u_{i}^{*}\right\|_{1, p}^{2}
$$

It means that for sufficiently small $\tau$, i.e., $\tau<\frac{2 C_{p}}{M^{2-p} C_{M} \lambda_{2}}, u_{i}=u_{i}^{*}$ holds.

Proposition 3.2. We assume (H3)' holds instead of (H3) in Theorem 3.1. Then the same results hold provided that $\tau<1 / C_{2}$. 
Proof. In the proof of Theorem 3.1, we have only used (H1)-(H2) in showing the existence of solution $u_{i} \in W_{0}^{1, p}(\Omega) \cap L^{\infty}(\Omega)(i=1,2, \ldots, N)$ for (DS). Hence, we are going to show the uniqueness using (H3)' . Let $u_{i}$ and $u_{i}^{*}$ be two solutions of (DS). Then, from (H3)', we have

$\int_{\Omega}\left(f\left(x, i \tau, u_{i}\right)-f\left(x, i \tau, u_{i}^{*}\right)\right)\left(u_{i}-u_{i}^{*}\right) d x \geq-C_{2} \int_{\Omega}\left(\beta\left(u_{i}\right)-\beta\left(u_{i}^{*}\right)\right)\left(u_{i}-u_{i}^{*}\right) d x$.

Applying the above inequality to (6), by the monotonicity of $-\Delta_{p}$ (the $p$ Laplacian operator),

$$
\left(1-\tau C_{2}\right) \int_{\Omega}\left(\beta\left(u_{i}\right)-\beta\left(u_{i}^{*}\right)\right)\left(u_{i}-u_{i}^{*}\right) d x \leq 0 .
$$

Then by (H1), if $\tau<1 / C_{2}$, we get $u_{i}=u_{i}^{*}$.

Now, we consider the bounds of $\left\{u_{i}\right\}(i=1,2, \ldots, N)$, which is constructed in Theorem 3.1 and Proposition 3.2 as solutions of (DS).

Theorem 3.3. We assume (H1)-(H2). Then there exist $C_{3}, C_{4}, C_{5}$, which are positive constants and independent of $\tau$, such that for all $i=1,2, \ldots, N$,

(a) $\left\|u_{i}\right\|_{\infty} \leq C_{3}$

(b) $\tau \sum_{i=1}^{m}\left\|u_{i}\right\|_{1, p}^{p} \leq C_{4}$,

(c) $\left\|\beta\left(u_{m}\right)\right\|_{2}^{2}+\sum_{i=1}^{m}\left\|\beta\left(u_{i}\right)-\beta\left(u_{i-1}\right)\right\|_{2}^{2} \leq C_{5}$,

where $m=1,2, \ldots, N$.

Proof. (a) Multiplying (DS) by $\left|\beta\left(u_{i}\right)\right|^{k} \beta\left(u_{i}\right)$ and integrating over $\Omega$, we have by (H2) and Hölder's inequality,

$$
\begin{aligned}
& \int_{\Omega}\left|\beta\left(u_{i}\right)\right|^{k} \beta\left(u_{i}\right) \beta\left(u_{i}\right) d x-\int_{\Omega} \tau \Delta_{p} u_{i}\left|\beta\left(u_{i}\right)\right|^{k} \beta\left(u_{i}\right) d x \\
\leq & \left\|\beta\left(u_{i}\right)\right\|_{k+2}^{k+1}\left\|\beta\left(u_{i-1}\right)\right\|_{k+2}+m(\Omega)^{1 /(k+2)} \tau C_{1}\left\|\beta\left(u_{i}\right)\right\|_{k+2}^{k+1} .
\end{aligned}
$$

Then

$$
\left\|\beta\left(u_{i}\right)\right\|_{k+2} \leq m(\Omega)^{1 / k+2} \tau C_{1}+\left\|\beta\left(u_{i-1}\right)\right\|_{k+2} .
$$

By induction, we have $\left\|\beta\left(u_{i}\right)\right\|_{k+2} \leq m(\Omega)^{1 /(k+2)} C_{1} T+\left\|\beta\left(u_{0}\right)\right\|_{k+2}$. Letting $k \rightarrow \infty,\left\|u_{i}\right\|_{\infty} \leq C\left(C_{1}, T, u_{0}\right)=: C_{3}$ by $(\mathrm{H} 1)$.

(b) Let $z \in W_{0}^{1, p}(\Omega) \cap L^{\infty}(\Omega)$ be fixed. Multiplying the equation (DS) by $u_{i}-z$ and integrating over $\Omega$, we have, by (H2),

$$
\begin{aligned}
& \left\langle\beta\left(u_{i}\right)-\beta\left(u_{i-1}\right), u_{i}\right\rangle-\left\langle\beta\left(u_{i}\right)-\beta\left(u_{i-1}\right), z\right\rangle+\tau|| u_{i} \|_{1, p}^{p} \\
\leq & \tau \int_{\Omega}\left|\nabla u_{i}\right|^{p-1}|\nabla z| d x+\tau C_{1} \int_{\Omega}\left|u_{i}-z\right| d x .
\end{aligned}
$$


Now, we apply Young's inequality to (7) to get

$$
\begin{aligned}
& \left\langle\beta\left(u_{i}\right)-\beta\left(u_{i-1}\right), u_{i}\right\rangle-\left\langle\beta\left(u_{i}\right)-\beta\left(u_{i-1}\right), z\right\rangle+\frac{\tau}{2}\left\|u_{i}\right\|_{1, p}^{p} \\
\leq & \left\{\tau\left(\frac{1}{2} \frac{p}{p-1}\right)^{-(p-1)}+2^{p} \lambda_{1} \tau^{2}\right\} \frac{1}{p}\|z\|_{1, p}^{p}+\tau C_{1}\left\|u_{i}\right\|_{\infty} m(\Omega) \\
& +\tau C_{1}\|z\|_{\infty} m(\Omega)+\frac{\tau^{2}}{p^{\prime}} C_{1}^{p^{\prime}} m(\Omega)+2^{p} \lambda_{1} \frac{\tau}{p} \sum_{j=1}^{i} \tau\left\|u_{j}\right\|_{1, p}^{p}
\end{aligned}
$$

for $i=1,2, \ldots, m$ and for arbitrary $m=1,2, \ldots, N$. By the property of the Legendre transform $\psi^{*}$ of $\psi=\int_{0}^{t} \beta(s) d s$,

$$
\int_{\Omega}\left(\psi^{*}\left(\beta\left(u_{i}\right)\right)-\psi^{*}\left(\beta\left(u_{i-1}\right)\right)\right) d x \leq \int_{\Omega}\left(\beta\left(u_{i}\right)-\beta\left(u_{i-1}\right)\right) u_{i} d x
$$

and by (8),

(9)

$$
\begin{aligned}
& \int_{\Omega}\left(\psi^{*}\left(\beta\left(u_{i}\right)\right)-\psi^{*}\left(\beta\left(u_{i-1}\right)\right)\right) d x-\left\langle\beta\left(u_{i}\right)-\beta\left(u_{i-1}\right), z\right\rangle+\frac{\tau}{2}\left\|u_{i}\right\|_{1, p}^{p} \\
\leq & \left\{\tau\left(\frac{1}{2} \frac{p}{p-1}\right)^{-(p-1)}+2^{p} \lambda_{1} \tau^{2}\right\} \frac{1}{p}\|z\|_{1, p}^{p}+\tau C_{1}\left\|u_{i}\right\|_{\infty} m(\Omega) \\
& +\tau C_{1}\|z\|_{\infty} m(\Omega)+\frac{\tau^{2}}{p^{\prime}} C_{1}^{p^{\prime}} m(\Omega)+2^{p} \lambda_{1} \frac{\tau}{p} \sum_{j=1}^{i} \tau\left\|u_{j}\right\|_{1, p}^{p}
\end{aligned}
$$

for $i=1,2, \ldots, m$. By summing (9) with respect to $i=1,2, \ldots, m$ and by (a),

$$
\begin{aligned}
& \int_{\Omega} \psi^{*}\left(\beta\left(u_{m}\right)\right)-\psi^{*}\left(\beta\left(u_{0}\right)\right) d x-\left\langle\beta\left(u_{m}\right)-\beta\left(u_{0}\right), z\right\rangle+\frac{\tau}{2} \sum_{i=1}^{m}\left\|u_{i}\right\|_{1, p}^{p} \\
\leq & C_{6}+C_{7} \tau \sum_{i=1}^{m} \sum_{j=1}^{i} \tau\left\|u_{j}\right\|_{1, p}^{p},
\end{aligned}
$$

where $C_{6}:=C\left(T, p, \lambda_{1}, C_{1}, C\left(C_{1}, T, u_{0}\right), m(\Omega),\|z\|_{\infty},\|z\|_{1, p}\right)$ and $C_{7}:=C\left(\lambda_{1}\right.$, $p$ ) and for $m=1,2, \ldots, N$. By $(10)$ and for arbitrary $\tau<\bar{\tau}=1 /\left(4 C_{7}\right)$,

$$
\begin{aligned}
& \int_{\Omega} \psi^{*}\left(\beta\left(u_{m}\right)\right) d x-\left\langle\beta\left(u_{m}\right), z\right\rangle+\frac{\tau}{4} \sum_{i=1}^{m}\left\|u_{i}\right\|_{1, p}^{p} \\
\leq & \int_{\Omega} \psi^{*}\left(\beta\left(u_{0}\right)\right) d x-\left\langle\beta\left(u_{0}\right), z\right\rangle+C_{6}+C_{7} \tau \sum_{i=1}^{m-1} \sum_{j=1}^{i} \tau\left\|u_{j}\right\|_{1, p}^{p} .
\end{aligned}
$$

Applying the discrete Gronwall's lemma to (11),

$$
\begin{aligned}
& \int_{\Omega} \psi^{*}\left(\beta\left(u_{m}\right)\right) d x-\left\langle\beta\left(u_{m}\right), z\right\rangle+\frac{\tau}{4} \sum_{i=1}^{m}\left\|u_{i}\right\|_{1, p}^{p} \\
\leq & C\left(\beta\left(u_{0}\right), T, p, \lambda_{1}, C_{1}, C\left(C_{1}, T, u_{0}\right), m(\Omega),\|z\|_{\infty},\|z\|_{1, p}\right) .
\end{aligned}
$$


DOUBLY NONLINEAR PARABOLIC EQUATIONS INVOLVING $p$-LAPLACIAN 1187

Hence by $\int_{\Omega} \psi^{*}\left(\beta\left(u_{m}\right)\right) d x-\left\langle\beta\left(u_{m}\right), z\right\rangle>-\infty$ and (12), $\tau \sum_{i=1}^{m}\left\|u_{i}\right\|_{1, p}^{p} \leq C_{4}$.

(c) Multiplying (DS) by $\beta\left(u_{i}\right)$ and integrating over $\Omega$, we get by (H2),

$$
\int_{\Omega}\left(\beta\left(u_{i}\right)-\beta\left(u_{i-1}\right)\right) \beta\left(u_{i}\right) d x \leq C_{1} \tau \int_{\Omega}\left|\beta\left(u_{i}\right)\right| d x .
$$

Using $a(a-b)=\frac{1}{2} a^{2}-\frac{1}{2} b^{2}+\frac{1}{2}(a-b)^{2}$,

$$
\left\|\beta\left(u_{i}\right)\right\|_{2}^{2}-\left\|\beta\left(u_{i-1}\right)\right\|_{2}^{2}+\left\|\beta\left(u_{i}\right)-\beta\left(u_{i-1}\right)\right\|_{2}^{2} \leq 2 C_{1} \tau\left\|\beta\left(u_{i}\right)\right\|_{1} .
$$

Summing the above inequality with respect to $i=1,2, \ldots, m$, we have by (a),

$$
\begin{aligned}
\left\|\beta\left(u_{m}\right)\right\|_{2}^{2}+\sum_{i=1}^{m}\left\|\beta\left(u_{i}\right)-\beta\left(u_{i-1}\right)\right\|_{2}^{2} & \leq 2 C_{1} \tau \sum_{i=1}^{m}\left\|\beta\left(u_{i}\right)\right\|_{1}+\left\|\beta\left(u_{0}\right)\right\|_{2}^{2} \\
& \leq 2 C_{1} T m(\Omega) C\left(C_{1}, T, u_{0}\right)+\left\|\beta\left(u_{0}\right)\right\|_{2}^{2}
\end{aligned}
$$

for $m=1,2, \ldots, N$. Thus $\left\|\beta\left(u_{m}\right)\right\|_{2}^{2}+\sum_{i=1}^{m}\left\|\beta\left(u_{i}\right)-\beta\left(u_{i-1}\right)\right\|_{2}^{2} \leq C_{5}$.

In the forthcoming discussion, the following notations will be used extensively. For vectors $u_{i}(i=0,1, \ldots, N)$ in Theorem 3.1 , we define two functions $u_{\tau}$ and $\bar{u}_{\tau}$ on $[0, T]$ by

$$
\begin{array}{ll}
u_{\tau}(0):=u_{0}, & u_{\tau}(t):=u_{i}+\frac{u_{i}-u_{i-1}}{\tau}(t-i \tau), \\
\bar{u}_{\tau}(0):=u_{0}, & \bar{u}_{\tau}(t):=u_{i},
\end{array}
$$

for $t \in((i-1) \tau, i \tau](i=1,2, \ldots, N)$ and $\tau=T / N$. Similarly, we define

$$
\begin{array}{ll}
v_{\tau}(0):=v_{0}, & v_{\tau}(t):=v_{i}+\frac{v_{i}-v_{i-1}}{\tau}(t-i \tau), \\
\bar{v}_{\mathcal{\tau}}(0):=v_{0}, & \bar{v}_{\tau}(t):=v_{i},
\end{array}
$$

where $v_{i}:=\beta\left(u_{i}\right)$ for $i=0,1, \ldots, N$. Also, we let $\bar{f}_{\tau}(t):=f_{i}=f\left(\cdot, i \tau, u_{i}\right)$ for $t \in((i-1) \tau, i \tau](i=1,2, \ldots, N)$.

Hence we can rewrite (DS) in a more compact form as

$$
\begin{aligned}
& v_{\tau}^{\prime}-\Delta_{p} \bar{u}_{\tau}+\bar{f}_{\tau}=0, \quad \text { a.e. in }[0, T] . \\
& \bar{v}_{\tau}=\beta\left(\bar{u}_{\tau}\right),
\end{aligned}
$$

\section{Estimates and limits}

In this section, we assume the hypotheses (H1)-(H4).

\subsection{Estimates}

First of all, by the consequence of Theorem 3.3, the followings are very easily proven.

$\bar{u}_{\tau}$ is bounded in $L^{p}\left(0, T ; W_{0}^{1, p}(\Omega)\right) \cap L^{\infty}\left(0, T ; L^{\infty}(\Omega)\right)$,

$\bar{v}_{\tau}$ is bounded in $L^{\infty}\left(0, T ; L^{2}(\Omega)\right)$. 
Moreover, by (14) and boundedness of $p$-Laplacian operator,

$$
-\Delta_{p} \bar{u}_{\tau} \text { is bounded in } L^{p^{\prime}}\left(0, T ; W^{-1, p^{\prime}}(\Omega)\right) .
$$

We emphasis that all the above boundedness are independent of $\tau$.

First of all, we have $u \in L^{p}\left(0, T ; W_{0}^{1, p}(\Omega)\right) \cap L^{\infty}\left(0, T ; L^{\infty}(\Omega)\right)$ which is a weak limit of $\bar{u}_{\tau}$ as $\tau \rightarrow 0$ by (14), i.e., $\bar{u}_{\tau}$ converges weakly to $u$ as $\tau \rightarrow 0$ in $L^{p}\left(0, T ; W_{0}^{1, p}(\Omega)\right) \cap L^{\infty}\left(0, T ; L^{\infty}(\Omega)\right)$. Moreover, by Theorem 3.3(c) we may use the term $w(x):=\sup _{t \in[0, T]} \frac{\partial \beta(u(x, t))}{\partial t}$ which is bounded in $L^{2}(\Omega)$ a.e.

Now, we consider the boundedness of $v_{\tau}^{\prime}$.

(a) We suppose $p \geq 2$. By (H3) and Theorem 3.3(c),

$$
\begin{aligned}
& \sum_{i=1}^{N} \int_{(i-1) \tau}^{i \tau}\left\|f(x, t, u)-f\left(x, t, \bar{u}_{\tau}\right)\right\|_{-1, p^{\prime}}^{p^{\prime}} d t \\
\leq & \sum_{i=1}^{N} \int_{(i-1) \tau}^{i \tau}\left\{\sup _{\|v\|_{1, p} \leq 1}\left(\int_{\Omega}|v(x)|^{p} d x\right)^{\frac{1}{p}}\left(\int_{\Omega}\left|f(x, t, u)-f\left(x, t, \bar{u}_{\tau}\right)\right|^{p^{\prime}} d x\right)^{\frac{1}{p^{\prime}}}\right\}^{p^{\prime}} d t \\
\leq & \lambda_{1}{ }^{p^{\prime}} C_{M} \tau \sum_{i=1}^{N} \int_{(i-1) \tau}^{i \tau}\left(\|u\|_{2}+\left\|\bar{u}_{\tau}\right\|_{2}\right)\|w\|_{2} d t \\
\leq & \lambda_{1}{ }^{p^{\prime}} C_{M} \tau\left(\|u\|_{L^{2}\left(0, T ; L^{2}(\Omega)\right)}+\left\|\bar{u}_{\tau}\right\|_{L^{2}\left(0, T ; L^{2}(\Omega)\right)}\right)\|w\|_{L^{2}\left(0, T ; L^{2}(\Omega)\right)} .
\end{aligned}
$$

And, by (H4),

$$
\begin{aligned}
& \sum_{i=1}^{N} \int_{(i-1) \tau}^{i \tau}\left\|f\left(x, t, \bar{u}_{\tau}(x, t)\right)-f\left(x, i \tau, \bar{u}_{\tau}(x, t)\right)\right\|_{-1, p^{\prime}}^{p^{\prime}} d t \\
\leq & \widetilde{C}_{M}^{p^{\prime}} \tau \sum_{i=1}^{N} \int_{(i-1) \tau}^{i \tau}\left[\sup _{\|v\|_{1, p} \leq 1}\left|\int_{\Omega}\right| v(x) \mid d x\right]^{1 / p^{\prime}} d t \\
\leq & \widetilde{C}_{M}^{p^{\prime}} \tau \sum_{i=1}^{N} \int_{(i-1) \tau}^{i \tau} \lambda_{4}^{p^{\prime}} d t=\widetilde{C}_{M}^{p^{\prime}} \tau \lambda_{4}^{p^{\prime}} T .
\end{aligned}
$$

Hence

$$
\begin{aligned}
& \left\|\bar{f}_{\tau}\left(x, t, \bar{u}_{\tau}\right)-f(x, t, u)\right\|_{\left.L^{p^{\prime}(0, T ; W}-1, p^{\prime}(\Omega)\right)}^{p^{\prime}} \\
\leq & 2^{p^{\prime}}\left(\lambda_{1}{ }^{p^{\prime}} C_{M} \tau\left(\|u\|_{L^{2}\left(0, T ; L^{2}(\Omega)\right)}+\left\|\bar{u}_{\tau}\right\|_{L^{2}\left(0, T ; L^{2}(\Omega)\right)}\right)\|w\|_{L^{2}\left(0, T ; L^{2}(\Omega)\right)}\right. \\
& \left.+\widetilde{C}_{M}^{p^{\prime}} \tau \lambda_{4}^{p^{\prime}} T\right) .
\end{aligned}
$$

(b) Now, we suppose $d^{*} \leq p<2$. In a very similar way, we have

$$
\begin{aligned}
& \left\|\bar{f}_{\tau}\left(x, t, \bar{u}_{\tau}\right)-f(x, t, u)\right\|_{L^{2}\left(0, T ; L^{2}(\Omega)\right)}^{2} \\
\leq & 2\left(\tau C_{M}\left(\|u\|_{L^{2}\left(0, T ; L^{2}(\Omega)\right)}+\left\|\bar{u}_{\tau}\right\|_{L^{2}\left(0, T ; L^{2}(\Omega)\right)}\right)\|w\|_{L^{2}\left(0, T ; L^{2}(\Omega)\right)}\right. \\
& \left.+T \widetilde{C}_{M}^{2} \tau m(\Omega)\right) .
\end{aligned}
$$


DOUBLY NONLINEAR PARABOLIC EQUATIONS INVOLVING $p$-LAPLACIAN 1189

Since $v_{\tau}^{\prime}=\Delta_{p} \bar{u}_{\tau}-\bar{f}_{\tau}$ in (13), by (16)-(18), we conclude that

$$
v_{\tau}^{\prime} \text { is bounded in } \begin{cases}L^{p^{\prime}}\left(0, T ; W_{0}^{-1, p^{\prime}}(\Omega)\right) & \text { if } \quad p \geq 2, \\ L^{2}\left(0, T ; L^{2}(\Omega)\right) & \text { if } \quad d^{*} \leq p<2 .\end{cases}
$$

\subsection{Limits}

(a) We suppose $p \geq 2$. As we mentioned in (14) and (15), we have $u$ and $v$ such that

$$
\begin{aligned}
& \bar{u}_{\tau} \rightarrow u \quad \text { weakly in } \quad L^{p}\left(0, T ; W_{0}^{1, p}(\Omega)\right) \cap L^{\infty}\left(0, T ; L^{\infty}(\Omega)\right), \\
& v_{\tau} \rightarrow v \quad \text { weakly star in } \quad W^{1, p^{\prime}}\left(0, T ; W^{-1, p^{\prime}}(\Omega)\right), \\
& v_{\tau} \rightarrow v \quad \text { weakly in } C\left(0, T ; L^{2}(\Omega)\right), \\
& \bar{v}_{\tau} \rightarrow v \quad \text { weakly star in } L^{p^{\prime}}\left(0, T ; W^{-1, p^{\prime}}(\Omega)\right), \\
& \bar{v}_{\tau} \rightarrow v \quad \text { strongly in } L^{2}\left(0, T ; L^{2}(\Omega)\right) .
\end{aligned}
$$

We note that the above sequences with $\tau$ are for some not relabeled subsequence. Also we note that

$$
\begin{aligned}
\lim _{\tau \rightarrow 0} \int_{0}^{T}\left\langle\phi, \bar{v}_{\tau}-v_{\tau}\right\rangle d t & \leq \lim _{\tau \rightarrow 0} \tau \sum_{i=1}^{N} \int_{(i-1) \tau}^{i \tau} \int_{\Omega} \phi(x, t)\left(\frac{v_{i}-v_{i-1}}{\tau}\right) d x d t \\
& =\lim _{\tau \rightarrow 0} \tau \sum_{i=1}^{N} \int_{(i-1) \tau}^{i \tau} \int_{\Omega} \phi(x, t) v_{\tau}^{\prime}(t) d x d t \\
& =\lim _{\tau \rightarrow 0} \tau \int_{0}^{T}\left\langle\phi, v_{\tau}^{\prime}\right\rangle d t=0
\end{aligned}
$$

for all $\phi \in L^{p}\left(0, T ; W_{0}^{1, p}(\Omega)\right)$ by $(19)$. Hence, $v_{\tau}$ and $\bar{v}_{\tau}$ have the same limit $v$ in $(21)$ and $(22)$.

(b) Now, we suppose $d^{*} \leq p<2$. In a very similar way, we have

$$
\begin{array}{lll}
\bar{u}_{\tau} \rightarrow u & \text { strongly in } & L^{2}\left(0, T ; L^{2}(\Omega)\right), \\
v_{\tau} \rightarrow v & \text { strongly in } & W^{1,2}\left(0, T ; L^{2}(\Omega)\right), \\
v_{\tau} \rightarrow v & \text { weakly in } \quad C\left(0, T ; L^{2}(\Omega)\right), \\
\bar{v}_{\tau} \rightarrow v & \text { strongly in } & L^{2}\left(0, T ; L^{2}(\Omega)\right) .
\end{array}
$$

Proof of Theorem 2.4. First of all, by (17) and (18), we have $f$ such that

(26) $\quad \bar{f}_{\tau} \rightarrow f \quad$ strongly in $\begin{cases}L^{p^{\prime}}\left(0, T ; W_{0}^{-1, p^{\prime}}(\Omega)\right) & \text { if } p \geq 2, \\ L^{2}\left(0, T ; L^{2}(\Omega)\right) & \text { if } d^{*} \leq p<2 .\end{cases}$ 
In addition, by (20), (22) [(23), (25), respectively] and (H1), we have $v=\beta(u)$. Therefore,

$$
-\Delta_{p} \bar{u}_{\tau} \rightarrow-\frac{\partial \beta(u)}{\partial t}-f \text { weakly in } \begin{cases}L^{p^{\prime}}\left(0, T ; W_{0}^{-1, p^{\prime}}(\Omega)\right) & \text { if } p \geq 2, \\ L^{2}\left(0, T ; L^{2}(\Omega)\right) & \text { if } d^{*} \leq p<2,\end{cases}
$$

from (13) since $\beta\left(\bar{u}_{\tau}(t)\right)=\bar{v}_{\tau}(t)$ for $t \in[0, T]$. And, by the property

$$
\int_{\Omega} \psi^{*}\left(\beta\left(u_{i}\right)\right)-\psi^{*}\left(\beta\left(u_{i-1}\right)\right) d x \leq \int_{\Omega}\left(\beta\left(u_{i}\right)-\beta\left(u_{i-1}\right)\right) u_{i} d x
$$

of the Legendre transform $\psi^{*}$ of $\psi=\int_{0}^{t} \beta(s) d s$,

$$
\begin{aligned}
& \limsup _{\tau \rightarrow 0} \int_{0}^{T}\left\langle-\Delta_{p} \bar{u}_{\tau}, \bar{u}_{\tau}\right\rangle d t \\
\leq & \limsup _{\tau \rightarrow 0} \sum_{i=1}^{N} \int_{(i-1) \tau}^{i \tau} \int_{\Omega} \frac{-\psi^{*}\left(\beta\left(u_{i}\right)\right)+\psi^{*}\left(\beta\left(u_{i-1}\right)\right)}{\tau} d x d t \\
& +\limsup _{\tau \rightarrow 0} \int_{0}^{T} \int_{\Omega}-\bar{f}_{\tau} \bar{u}_{\tau} d x d t .
\end{aligned}
$$

Moreover, since $\psi^{*}(\beta(u(i \tau)))-\psi^{*}(\beta(u((i-1) \tau)))=\frac{\partial \psi^{*}}{\partial s}(\beta(u(\sigma)))(i \tau-(i-1) \tau)$ for $\sigma \in((i-1) \tau, i \tau]$,

$$
\begin{aligned}
& \limsup _{\tau \rightarrow 0} \int_{0}^{T}\left\langle-\Delta_{p} \bar{u}_{\tau}, \bar{u}_{\tau}\right\rangle d t \\
\leq & \limsup _{\tau \rightarrow 0} \sum_{i=1}^{N} \int_{(i-1) \tau}^{i \tau} \frac{1}{\tau} \int_{\Omega}-\tau \frac{\partial \psi^{*}(\beta(u(s)))}{\partial s} d x d t+\int_{0}^{T}\langle-f, u\rangle d t \\
= & \int_{0}^{T} \int_{\Omega}-\frac{\partial \psi^{*}(\beta(u(s)))}{\partial s} d x d t+\int_{0}^{T}\langle-f, u\rangle d t
\end{aligned}
$$

by $(20)$ and $(26)\left[(23),(26)\right.$, respectively]. Also, since $\psi(t)=\int_{0}^{t} \beta(s) d s, \psi^{\prime}(t)=$ $\beta(t)$ and $\left(\psi^{*}\right)^{\prime}=\left(\psi^{\prime}\right)^{-1}$,

$$
\begin{aligned}
& \limsup _{\tau \rightarrow 0} \int_{0}^{T}\left\langle-\Delta_{p} \bar{u}_{\tau}, \bar{u}_{\tau}\right\rangle d t \\
\leq & \int_{0}^{T} \int_{\Omega}-\frac{\partial \beta(u(s))}{\partial s} u(s) d x d t+\int_{0}^{T}\langle-f, u\rangle d t \\
= & \int_{0}^{T}\left\langle-\frac{\partial \beta}{\partial t}-f, u\right\rangle d t .
\end{aligned}
$$

Therefore, by Lemma 2.1 (Minty's Theorem), (27) and (28), there exists a unique solution $u$ of (1) such that

$$
\begin{cases}u \in L^{p}\left(0, T ; W_{0}^{1, p}(\Omega)\right) \cap L^{\infty}\left(0, T ; L^{\infty}(\Omega)\right) & \text { if } \quad p \geq 2 \\ u \in L^{2}\left(0, T ; L^{2}(\Omega)\right) & \text { if } \quad d^{*} \leq p<2 .\end{cases}
$$


Remark 4.1. We may consider more generalized equation of the form

$$
\begin{cases}\frac{\partial \beta(u)}{\partial t}-\operatorname{div} a(x, u, D u)+f(x, t, u)=0 & \text { in } \Omega \times[0, T], \\ u=0 & \text { on } \partial \Omega \times[0, T], \\ u(\cdot, 0)=u^{0} & \text { in } \Omega,\end{cases}
$$

where the nonlinear operator $-\operatorname{div} a(x, u, D u)$ is same as the operator in Lemma 2.2. As we mentioned in the proof of Theorem 3.1, since the $p$-Laplacian operator $\Delta_{p} u=\operatorname{div}\left(|\nabla u|^{p-2} \nabla u\right)(1<p<\infty)$ is a special case of $-\operatorname{div} a(x, u, D u)$, the above equation has the same results provided we assume the conditions (H1) $-(\mathrm{H} 4)$ and $(\mathrm{H} 3)^{\prime}$.

\section{References}

[1] R. Adams, Sobolev Spaces, Academic Press, New York, 1975.

[2] V. Barbu, Nonlinear Semigroups and Differential Equations in Banach Spaces, Noordhoff Internat. Publ., Leyden, 1976.

[3] A. Bensoussan, L. Boccardo, and F. Murat, On a nonlinear partial differential equation having natural growth terms and unbounded solution, Ann. Inst. H. Poincaré Anal. Non Linéaire 5 (1988), no. 4, 347-364.

[4] J. I. Diaz and J. F. Padial, Uniqueness and existence of solutions in the $B V_{t}(Q)$ space to a doubly nonlinear parabolic problem, Differential Integral Equations 40 (1996), no. 2, 527-560.

[5] A. Eden, B. Michaux, and J. M. Rakotoson, Semi-discretized nonlinear evolution equations as discrete dynamical systems and error analysis, Indiana Univ. Math. J. 39 (1990), no. 3, 737-783.

[6] Doubly nonlinear parabolic type equations as dynamical systems, J. Dynam. Differential Equations 3 (1991), no. 1, 87-131.

[7] M. Efendiev and A. Miranville, New models of Cahn-Hilliard-Gurtin equations, Contin. Mech. Thermodyn. 16 (2004), no. 5, 441-451.

[8] A. El Hachimi and H. El Ouardi, Existence and regularity of a global attractor for doubly nonlinear parabolic equations, Electron. J. Differential Equations 2002 (2002), no. 45, $1-15$.

[9] M. Gurtin, Generalized Ginzburg-Landau and Cahn-Hilliard equations based on a microforce balance, Phys. D. 92 (1996), no. 3-4, 178-192.

[10] A. G. Kartasatos and M. E. Parrott, The weak solution of a functional-differential equation in a general Banach space, J. Differential Equations 75 (1988), no. 2, 290-302.

[11] V. Le and K. Schmit, Global Bifurcation in Variational Inequalities, Springer-Verlag, New York, 1997.

[12] N. Merazga and A. Bouziani, On a time-discretization method for a semilinear heat equation with purely integral conditions in a nonclassical function space, Nonlinear Analysis 66 (2007), no. 3, 604-623.

[13] A. Miranville and G. Schimperna, Global solution to a phase transition model based on a microforce balance, J. Evol. Equ. 5 (2005), no. 2, 253-276.

[14] F. Otto, $L^{1}$-contraction and uniqueness for quasilinear elliptic-parabolic equations, J. Differential Equations 131 (1996), no. 1, 20-38.

[15] A. Rougirel, Convergence to steady state and attractors for doubly nonlinear equations, J. Math. Anal. Appl. 339 (2008), no. 1, 281-294.

[16] J. M. Rakotoson, On some degenerate and nondegenerate quasilinear elliptic systems with nonhomogeneous Dirichlet boundary condition, Nonlinear Analysis 13 (1989), no. $2,165-183$ 
[17] M. Schatzman, Stationary solutions and asymptotic behavior of a quasilinear degenerate parabolic equation, Indiana Univ. Math. J. 33 (1984), no. 1, 1-29.

[18] R. E. Showalter, Monotone operators in Banach space and nonlinear partial differential equations, Mathematical Surveys and Monographs 49, AMS, 1997.

\section{KIYEON SHIN}

Department of Mathematics

Pusan National University

Pusan 609-735, Korea

E-mail address: kyshin@pusan.ac.kr

SUJIN KANG

Department of Nanomaterials Engineering

Pusan National University

PUSAN 609-735, Korea

E-mail address: sjnisj@pusan.ac.kr 